\title{
Uso de líquido ruminal en agua de bebida de pollos broiler criados en condiciones de altura
}

\author{
Use of ruminal fluid in drinking water of broilers raised in high altitude conditions \\ Jorge Isaac Castro Bedriñana ${ }^{1,2}$, Doris Maritza Chirinos Peinado ${ }^{1}$, \\ William Nolberto Sierra Rojas ${ }^{1}$
}

\section{Resumen}

La investigación se realizó en una granja ubicada en la sierra central del Perú, a 3270 msnm, para determinar el efecto del uso de niveles de líquido ruminal en el agua de bebida de pollos broilers. Se utilizaron 200 pollos Cobb desde el primer día de edad. Las aves fueron distribuidas en cuatro grupos de 50, en iguales proporciones de machos y hembras. Los tratamientos fueron: T1: Agua de bebida sola (control); T2: 87.5\% de agua de bebida $+12.5 \%$ de líquido ruminal; $\mathrm{T} 3: 75 \%$ de agua de bebida $+25 \%$ de líquido ruminal; T4: $62.5 \%$ de agua de bebida $+37.5 \%$ de líquido ruminal. Los pollos fueron alimentados con un concentrado de 'inicio' durante tres semanas. La inclusión de líquido ruminal en el agua de bebida mejoró la ganancia de peso y la conversión alimenticia $(\mathrm{p}<0.01)$. El empleo de $37.5 \%$ de líquido ruminal en el agua de bebida permitió tener $19.4 \%$ más de ganancia de peso y $15.5 \%$ más de conversión alimenticia.

Palabras clave: líquido ruminal; glucanasas; celulasas; fitasas; conversión alimenticia; aves de recría

\section{Abstract}

The study was carried out in a farm located in the central highlands of Peru, at 3270 $\mathrm{m}$ above the sea level, to determine the effect of the use of three ruminal fluid levels in the drinking water of broilers. Two hundred Cobb chickens were used from the first day of age. The chicks were distributed in four groups of 50, in equal proportions of males and females. The treatments were: T1: drinking water alone (control); T2: 87.5\% drinking water $+12.5 \%$ ruminal fluid; T3: $75 \%$ drinking water $+25 \%$ ruminal fluid; T4: $62.5 \%$

\footnotetext{
${ }^{1}$ Facultad de Zootecnia, Universidad Nacional del Centro del Perú, Huancayo, Perú

${ }^{2}$ E-mail: jorgecastrobe@yahoo.com
}

Recibido: 6 de febrero de 2017

Aceptado para publicación: 11 de mayo de 2018 
drinking water $+37.5 \%$ ruminal fluid. The chicks were fed with a starter diet for three weeks. The inclusion of ruminal fluid in drinking water improved weight gain and feed conversion $(p<0.01)$. The use of $37.5 \%$ ruminal fluid in drinking water allowed a $19.4 \%$ increase in weight gain and a $15.5 \%$ increase in feed conversion.

Key words: ruminal fluid; glucanases; cellulases; phytase; feed conversion; poultry

\section{INTRODUCCIÓN}

En la alimentación de pollos broiler, se reporta que el uso de dietas conteniendo ingredientes ricos en polisacáridos complejos no amiláceos afectan la digestibilidad de los nutrientes (Pinheiro et al., 2008; Singh et al., 2010; Amerah, 2015), debido al vínculo entre la estructura de la pared celular y diversos nutrientes (Bach Knudsen, 2001; Kim et al., 2004) y por la propiedad de la fibra de aumentar la viscosidad de la digesta (Choct et al., 1995).

Los polisacáridos no amiláceos como celulosa, arabinoxilanos, inulina, quitinas, pectinas, beta-glucanos y lignina, presentes en la pared celular de plantas (Bach Knudsen, 2001) son insuficientemente digeridos por las aves de corral, especialmente en aves jóvenes (Batal y Parsons, 2002, 2004; Zelenka y Ceresnakova, 2005).

La flora microbiana de las aves alberga microorganismos que cumplen un papel importante en los procesos digestivos y de protección frente a los agentes patógenos (Waite y Taylor, 2014); sin embargo, las aves no están capacitadas para utilizar adecuadamente las dietas vegetales ricas en fibra (Svihus et al., 2005; Tester et al., 2006; Singh et al., 2010), por lo que se ha evaluado el uso de fitasas (Gizzi et al., 2008; Brask-Pedersen et al., 2011, 2013) y complejos multienzimáticos a base de celulasa, xilanasa, â-glucanasa, amilasa, proteasa, pectinasa y lipasa, entre otras (Jiang et al., 2008; Olukosi et al., 2008; Murphy et al., 2009; Amerah et al., 2009; Svihus, 2014; Dhama et al., 2014), a fin de mejorar el incremento de peso, la eficiencia alimenticia y la retención de fósforo fítico y fósforo total, entre otros minerales y nutrientes esenciales.

Si bien este tipo de aditivos están disponibles en el mercado (Biovet, 2015), pueden ser sustituidos por otras fuentes naturales ricas en principios bioactivos (Castro y Chirinos, 2007), como el líquido ruminal, que es desechado a los ríos y desagües ocasionando problemas de contaminación (PMAR, 2004) $\mathrm{y}$, de esa forma, se desperdicia una materia prima de posible reutilización para la alimentación animal.

El rumen es una cámara simbiótica de fermentación anaeróbica que permite transformar la masa vegetal indigestible en productos alimenticios de calidad (Stevenson y Weimer, 2007; Welkie et al., 2009; Sundset et al., 2009; Jami y Mizrahi, 2012). El tipo de población microbiana responde al tipo de dieta (Santschi et al., 2005; Saro et al., 2014; Kim et al., 2014).

En este sentido, el líquido ruminal sería una importante fuente de nutrientes y otros compuestos activos que permitirían una mejor respuesta productiva de los pollos, mejorando la hidrólisis de fracciones fibrosas, del ácido fítico y de otras sustancias complejas de difícil digestión por los monogástricos; más aún, considerando que en las primeras semanas de vida tienen un sistema digestivo inmaduro (Batal y Parsons, 2002, 2004; Krás et $a l ., 2013)$. Es así que el objetivo de este estudio fue determinar el efecto del uso de diferentes niveles de líquido ruminal en el agua de bebida y medir su efecto en el comportamiento productivo de pollos broiler. 


\section{Materiales y Métodos}

El experimento se llevó a cabo en una granja ubicada en la provincia de Huancayo, departamento de Junín, Perú, a 3200 msnm. Se trabajó con 200 pollos BB sexados de la Línea Cobb (100 machos y 100 hembras), distribuidos aleatoriamente en cuatro grupos de 50 pollos ( 25 machos y 25 hembras): T1: agua de bebida sola (control); T2: $87.5 \%$ de agua de bebida $+12.5 \%$ de líquido ruminal; T3: $75 \%$ de agua de bebida $+25 \%$ de líquido ruminal; $\mathrm{T} 4: 62.5 \%$ de agua de bebida $+37.5 \%$ de líquido ruminal, ofrecida diariamente por un periodo de 21 días.

En la formulación del concentrado de inicio se utilizó maíz amarillo, harina de pescado Prime, torta de soya, soya integral, repaso de maíz, afrecho de trigo, polvillo de arroz y DL-metionina, considerando un aporte de $21 \%$ de proteína total y $3100 \mathrm{kcal} \mathrm{EM} / \mathrm{kg}$, $5 \%$ de fibra cruda, $1.0 \%$ de calcio, $0.45 \%$ de fósforo disponible, $0.42 \%$ de metionina, $1.05 \%$ de lisina y $0.21 \%$ de triptófano (NRC, 1994). $\mathrm{El}$ análisis proximal del alimento, realizado en el Laboratorio de Nutrición de la Facultad de Zootecnia de la Universidad Nacional del Centro del Perú, reportó $10.77 \%$ de humedad. En base seca, los contenidos de proteína, fibra, extracto etéreo, ceniza y extracto libre de nitrógeno fueron 20.61, 5.23, 2.96, 6.16 y $65.04 \%$, respectivamente.

El contenido ruminal fue colectado diariamente en el camal de Chupaca (a 30 minutos de Huancayo), en recipientes de acero quirúrgico con tapa $(7,10$ y 21 litros diarios durante la primera, segunda y tercera semana, respectivamente). El líquido ruminal fue filtrado con gasa, agregando el líquido ruminal al agua de bebida en las proporciones previstas y ofrecido en bebederos de volteo. Tanto el alimento concentrado y el agua de bebida fueron ofrecidos ad libitum. El consumo de concentrado fue registrado semanalmente, mientras que el consumo de agua de bebida fue registrado diariamente empleándose probetas graduadas, obteniéndose por dife- rencia entre la cantidad ofrecida y la cantidad residual. El peso vivo individual de los pollos fue registrado al inicio y cada 7 días hasta los 21 días de edad. El peso se midió en una balanza digital electrónica de $1 \mathrm{~g}$ con 5 kg de capacidad.

Los casos de mortalidad fueron registrados. Los datos colectados fueron analizados estadísticamente mediante un diseño de bloques completos al azar, realizándose el análisis de variancia para los pesos vivos e incrementos de peso. El consumo de alimento y conversión alimenticia fue determinado como promedio por tratamiento. Para el procesamiento estadístico de los datos se empleó el programa estadístico SPSS v.12.

\section{Resultados y Discusión}

Los tratamientos con diferente composición del agua de bebida afectaron en forma significativa el comportamiento productivo de los pollos en el periodo de 1 a 21 días de edad (cuadros 1 y 2).

El efecto positivo del líquido ruminal en el agua de bebida se pudo observar desde la primera semana de edad $(\mathrm{p}<0.01)$. Esta tendencia fue sostenida hasta los 21 días, donde se observa el mayor peso vivo de los pollos al incluir $37.5 \%$ de líquido ruminal en el agua de bebida. Estos resultados dan evidencia científica del efecto positivo de incluir líquido ruminal en el agua de bebida a pollos broilers alimentados con dietas a base de ingredientes vegetales ricos en carbohidratos complejos, como el subproducto de trigo, polvillo de arroz y repaso de maíz.

No se dispone de referencias específicas por el uso de líquido ruminal en broilers; sin embargo, el empleo de suplementos enzimáticos como las fitasas (Gizzi et al., 2008) y de complejos multienzimáticos a base de celulasa, xilanasa, $\beta$-glucanasa, amilasa, proteasa, pectinasa, lipasa, entre otros, reportan mejoras en las ganancias de peso, efi- 
Cuadro 1. Peso vivo promedio de pollos BB Cobb (g) hasta los 21 días de edad por tratamiento (proporción de líquido ruminal en el agua de bebida), según sexo

\begin{tabular}{|c|c|c|c|c|c|}
\hline \multirow{2}{*}{ Peso } & \multicolumn{4}{|c|}{ Tratamientos $^{1}$} & \multirow{2}{*}{ Promedio } \\
\hline & $\mathrm{T} 1$ & $\mathrm{~T} 2$ & $\mathrm{~T} 3$ & $\mathrm{~T} 4$ & \\
\hline \multicolumn{6}{|l|}{ Inicial $^{2}$} \\
\hline Machos & 46.4 & 47.4 & 47.4 & 47.8 & $47.3^{\mathrm{a}}$ \\
\hline Hembras & 47.3 & 45.8 & 47.2 & 46.2 & $46.6^{\mathrm{a}}$ \\
\hline Promedio & $46.8^{\mathrm{a}}$ & $46.6^{\mathrm{a}}$ & $47.3^{\mathrm{a}}$ & $47.0^{\mathrm{a}}$ & 47.0 \\
\hline \multicolumn{6}{|l|}{1 semana } \\
\hline Machos & 87.0 & 111.8 & 105.1 & 107.4 & $102.8^{\mathrm{a}}$ \\
\hline Hembras & 85.6 & 110.2 & 100.0 & 100.0 & $98.9^{\mathrm{b}}$ \\
\hline Promedio & $86.3^{\mathrm{c}}$ & $111.0^{\mathrm{a}}$ & $102.6^{\mathrm{b}}$ & $103.5^{\mathrm{b}}$ & 100.9 \\
\hline \multicolumn{6}{|l|}{2 semanas } \\
\hline Machos & 208.6 & 222.6 & 220.9 & 227.8 & $219.9^{a}$ \\
\hline Hembras & 212.4 & 230.7 & 231.3 & 246.7 & $230.3^{\mathrm{b}}$ \\
\hline Promedio & $210.5^{\mathrm{b}}$ & $226.4^{\mathrm{a}}$ & $226.1^{\mathrm{a}}$ & $237.3^{\mathrm{a}}$ & 225.1 \\
\hline \multicolumn{6}{|l|}{3 semanas } \\
\hline Machos & 338.6 & 355.3 & 356.4 & 384.3 & $358.7^{\mathrm{b}}$ \\
\hline Hembras & 342.9 & 374.9 & 394.6 & 411.7 & $381.0^{\mathrm{a}}$ \\
\hline Promedio & $340.8^{\mathrm{c}}$ & $365.1^{\mathrm{b}}$ & $375.5^{\mathrm{b}}$ & $398.0^{\mathrm{a}}$ & 369.8 \\
\hline
\end{tabular}

Cuadro 2. Incremento de peso de pollos BB Cobb (g) a la tercera semana de edad por tratamiento (proporción de líquido ruminal en el agua de bebida), según sexo

\begin{tabular}{cccccc}
\hline & \multicolumn{4}{c}{ Tratamientos $^{1}$} & \multirow{2}{*}{ Promedio } \\
\cline { 2 - 5 } & $\mathrm{T} 1$ & $\mathrm{~T} 2$ & $\mathrm{~T} 3$ & $\mathrm{~T} 4$ & \\
\hline Machos & 292.2 & 307.8 & 309.0 & 336.5 & $311.4^{\mathrm{b}}$ \\
Hembras & 295.6 & 329.1 & 347.4 & 365.4 & $334.4^{\mathrm{a}}$ \\
\hline Promedio & $293.9^{\mathrm{c}}$ & $318.5^{\mathrm{b}}$ & $328.2^{\mathrm{b}}$ & $351.0^{\mathrm{a}}$ & 322.9 \\
\hline a,b,c & Superíndices diferentes entre filas (promedios por tratamientos) y entre columnas (promedios \\
entre sexos) son significativamente diferentes ( $\mathrm{p}<0.01$ ) \\
${ }^{1}$ T1: 100\% agua; T2: 87.5\% agua - 12.5\% líquido ruminal; T3: 75\% agua - 25\% líquido ruminal; T4: \\
62.5\% agua - 37.5\% líquido ruminal
\end{tabular}


Cuadro 3. Consumo de alimento, consumo de materia seca y conversión alimenticia de pollos BB Cobb en el periodo 1-21 días de edad, según tratamiento (proporción de líquido ruminal en el agua de bebida)

\begin{tabular}{lcccc}
\hline & \multicolumn{4}{c}{ Tratamientos $^{1}$} \\
\cline { 2 - 5 } & $\mathrm{T} 1$ & $\mathrm{~T} 2$ & $\mathrm{~T} 3$ & $\mathrm{~T} 4$ \\
\hline Consumo de alimento $(\mathrm{g})$ & 597 & 611 & 600 & 601 \\
Consumo de materia seca $(\mathrm{g})$ & 532 & 545 & 535 & 536 \\
Conversión alimenticia - CA & $1.81^{\mathrm{a}}$ & $1.71^{\mathrm{ab}}$ & $1.63^{\mathrm{bc}}$ & $1.53^{\mathrm{c}}$ \\
Relación porcentual de la CA & 100.0 & 94.5 & 90.1 & 84.5 \\
Porcentaje de mejora de la CA & & 5.5 & 9.9 & 15.5 \\
\hline
\end{tabular}

$\mathrm{a}, \mathrm{b}, \mathrm{c}$ Superíndices diferentes dentro de filas indican diferencia significativa $(\mathrm{p}<0.01)$

${ }^{1}$ T1: $100 \%$ agua; T2: $87.5 \%$ agua $-12.5 \%$ líquido ruminal; T3: $75 \%$ agua $-25 \%$ líquido ruminal; T4: $62.5 \%$ agua $-37.5 \%$ líquido ruminal

Cuadro 4. Consumo de líquido, agua y líquido ruminal (en litros) por pollos BB Cobb, según tratamiento (proporción de líquido ruminal en el agua de bebida)

\begin{tabular}{lcccc}
\hline & \multicolumn{4}{c}{ Tratamientos $^{1}$} \\
\cline { 2 - 5 } & $\mathrm{T} 1$ & $\mathrm{~T} 2$ & $\mathrm{~T} 3$ & $\mathrm{~T} 4$ \\
\hline Primera semana & 0.189 & 0.259 & 0.238 & 0.205 \\
Segunda semana & 0.335 & 0.371 & 0.360 & 0.357 \\
Tercera semana & 0.906 & 0.941 & 0.940 & 0.937 \\
Consumo acumulativo & 1.429 & 1.571 & 1.538 & 1.498 \\
Consumo exclusivo de agua & 1.429 & 1.375 & 1.154 & 0.936 \\
Consumo de líquido ruminal & & 0.196 & 0.384 & 0.562 \\
\hline
\end{tabular}

${ }^{1}$ T1: $100 \%$ agua; T2: $87.5 \%$ agua $-12.5 \%$ líquido ruminal; T3: 75\% agua - 25\% líquido ruminal; T4: $62.5 \%$ agua $-37.5 \%$ líquido ruminal

ciencia alimenticia y retención de fósforo fítico y fósforo total (Cowieson et al., 2010; Jiang et al., 2008; Olukosi et al., 2008; Murphy et al., 2009; Amerah et al., 2009; Svihus, 2014; Dhama et al., 2014).

Si bien en el presente estudio no se emplearon aditivos enzimáticos comerciales ni aditivos nutricionales, el líquido ruminal adicionado al agua de bebida indicaría una mejor utilización de la energía que se encuentra en los carbohidratos complejos no solubles como la celulosa, pentosanos, $\beta$-glucanos y oligopolisacáridos (Gracia et al., 2003; Krás et al., 2013), que las enzimas propias del pollo no las pueden digerir (Batal y Parsons, 2004; Zelenka y Ceresnakova, 2005). Asimismo, el líquido ruminal estaría contribuyendo con vitaminas del complejo B, vitamina $\mathrm{K}$, aminoácidos libres y otros nutrientes 
Cuadro 5. Ecuaciones de regresión para el incremento de peso y conversión alimenticia con respecto al nivel de líquido ruminal (JR) (variable dependiente) utilizado en el agua de bebida ofrecida a pollos BB Cobb durante 21 días

\begin{tabular}{lcccc}
\hline $\begin{array}{l}\text { Variable } \\
\text { independiente }\end{array}$ & Ecuaciones de regresión & $\begin{array}{c}\text { Coeficiente de } \\
\text { determinación } \\
\left(\mathrm{R}^{2}\right)\end{array}$ & $\begin{array}{c}\text { Coeficiente de } \\
\text { correlación } \\
\text { Pearson }(\mathrm{R})\end{array}$ & $\begin{array}{c}\text { Valor } \\
\mathrm{de} \mathrm{p}\end{array}$ \\
\hline $\begin{array}{l}\text { Incremento de } \\
\text { peso }\end{array}$ & $\mathrm{Y}=295.78+1.446(\% \mathrm{LR})$ & 0.689 & 0.83 & 0.011 \\
$\begin{array}{l}\text { Conversión } \\
\text { alimenticia }\end{array}$ & $\mathrm{Y}=1.805-0.0074(\% \mathrm{LR})$ & 0.938 & 0.97 & 0.000 \\
\hline
\end{tabular}

(Santschi, 2005; Rakotoarivonina et al., 2014); y adicionalmente, la hidrolisis del ácido fítico incrementa la disponibilidad de fósforo, zinc y otros nutrientes esenciales que estaban ligados al ácido fítico (Gizzi et al., 2008). Esto habría permitido mejorar las ganancias de peso a los 21 días de la crianza $(\mathrm{p}<0.01)$.

Se reporta que numerosas enzimas han sido aisladas a partir de varias especies bacterianas del rumen que degradan a los polímeros de las paredes celulares de las plantas, mientras otras degradan las toxinas presentes en el grupo de las especies vegetales, entre ellas, las taninasas (Gonzales, 2004). La fitasa es también producida por los hongos Saccharomyces cerevisiae y Asper-gillus, bacterias Pseudomonas y Bacillus subtilis, levaduras y por algunos microorga-nismos del rumen (Camiruaga, 2001). Los microorganismos del rumen también sintetizan vitaminas, sobre todo del complejo B y la vitamina $\mathrm{K}_{2}$ (Montañez et al., 2006); enzimas y nutrientes que en el presente estudio demostraron su eficacia.

El consumo promedio de alimento por pollo a los 21 días de la crianza, tanto en fresco como en base seca, fue similar en los cuatro tratamientos (Cuadro 3), pero su utilización fue mejorada por la inclusión del líquido ruminal $(\mathrm{p}<0.01)$. Es así que la conversión alimenticia fue mejorada en $15 \%$ al utilizar $37.5 \%$ de líquido ruminal en el agua de bebida. Estos resultados son superiores a los valores reportados con el uso de promotores de crecimiento o complejos enzimáticos, donde se indican mejoras entre 3 y $6 \%$ en la eficiencia alimenticia (Luna et al., 2010; Tupayachi et al., 2016). Esta alternativa de reutilización de productos de desecho contribuye adicionalmente con la sostenibilidad del sistema, pues parte del líquido ruminal puede ser reutilizado a fin de disminuir la contaminación ambiental (PMAR, 2004).

El consumo promedio de líquido por pollo por día, hasta los 21 días, estuvo entre 68 a $75 \mathrm{ml}$, y los consumos promedio por día de líquido ruminal fueron de $9.3,18.3$ y $26.8 \mathrm{ml} /$ día para los tratamientos que tuvieron 12.5, 25.0 y $37.5 \%$ de líquido ruminal (Cuadro 4 ).

La influencia del nivel de líquido ruminal incluido en el agua de bebida sobre el incremento de peso y conversión alimenticia hasta los 21 días fue altamente significativa (Cuadro 5). El coeficiente de regresión para el incremento de peso indica que por cada unidad porcentual de líquido ruminal se incrementa la ganancia de peso en $1.45 \mathrm{~g}$; asimismo, el coeficiente de determinación indica que el $68.9 \%$ de la variabilidad observa- 
da en el incremento de peso sería atribuible al nivel de líquido ruminal utilizado; resultado que ratifica el efecto positivo del líquido ruminal sobre las ganancias de peso.

Similarmente, el coeficiente de regresión del efecto del contenido de líquido ruminal en el agua de bebida sobre la conversión alimenticia hasta los 21 días indica que por cada unidad porcentual de líquido ruminal utilizado el valor de la conversión alimenticia disminuye en 0.007 , en tanto que el coeficiente de determinación indica que el $93.8 \%$ de la variabilidad observada en la conversión alimenticia puede ser atribuida al nivel de líquido ruminal incluido en el agua de bebida.

La mortalidad fue de 10.0, 8.0, $4.0 \mathrm{y}$ $4.0 \%$ para los tratamientos $\mathrm{T} 1, \mathrm{~T} 2, \mathrm{~T} 3 \mathrm{y} \mathrm{T} 4$, respectivamente, lo cual sería indicativo adicional de una actividad positiva del líquido ruminal en la salud de los pollos, debiéndose realizar estudios específicos sobre este aspecto. Los casos de mortalidad ocurrieron entre los 12 y 20 días de edad, debido al síndrome de mal de altura, determinado por la necropsia correspondiente.

\section{Conclusión}

El líquido ruminal es una fuente alimenticia no convencional utilizable en la alimentación de pollos que mejora la respuesta productiva al ser incluida en el agua de bebida.

\section{Literatura Citada}

\section{Amerah AM, Ravindran $V$, Lentle RG}

2009. Influence of wheat hardness and xylanase supplementation on the energy utilisation, digestive tract development and digesta parameters of broiler starters. Anim Prod Sci 49: 71-78. doi: 10.1071/ EA08162
2. Amerah AM. 2015. Interactions between wheat characteristics and feed enzyme supplementation in broiler diets. Review article. Anim Feed Sci Tech 199: 1-9. doi: 10.1016/j.anifeedsci.2014.09.012

3. Bach Knudsen KE. 2001. The nutritional significance of «dietary fibre» analysis. Anim Feed Sci Tech 90: 3-20. doi: 10.1016/S0377-8401(01)00193-6

4. Batal AB, Parsons CM. 2002. Effects of age on nutrient digestibility in chicks fed different diets. Poult Sci 81:400-407. doi: $10.1093 / \mathrm{ps} / 81.3 .400$

5. Batal AB, Parsons CM. 2004. Utilization of various carbohydrate sources as affected by age in the chick. Poult Sci 83: 1140-1147.

6. Biovet SA. 2015. Productos enzimáticos para aves y cerdos: Alquerzima, Alquerzim aviar, Alquerzim aviar Plus, Alquerzom crisociolida aviar, Alquerzim fat. [Internet]. Disponible en http:// www.biovet-alquermes.com/index.php/ enzimaticos

7. Brask-Pedersen DN, Glitso LV, Skov LK, Lund P, Sehested J. 2011. Effect of exogenous phytase on feed inositol phosphate hydrolysis in an in vitro rumen fluid buffer system. J Dairy Sci 94: 951959. doi: 10.3168/jds.2010-3504

8. Brask-Pedersen DN, Glitso LV, Skov LK, Lund P, Sehested J. 2013. Effect of exogenous phytase on degradation of inositol phosphate in dairy cows. J Dairy Sci 96: 1691-1700. doi: 10.3168/jds.20115278

9. Camiruaga, M, Garcia F, Elera R, Y Simonetti C. 2001. Respuesta productiva de pollos broilers a la adición de enzimas exógenas a dietas basadas en maíz o triticale. Cien Inv Agr 28(1): 23 36.

10. Castro J, Chirinos D. 2007. Manual de formulación de raciones balanceadas para animales. Lima: CONCYTEC. 219 p.

11. Choct M, Hughes RJ, Trimble RP, Angkanaporn K, Annison G. 1995. Non-starch polysaccharide-degrading 
enzymes increase the performance of broiler chickens fed wheat of low apparent metabolizable energy. J Nutr 125: 485-492.

12. Cowieson, AJ, Bedford MR, Ravindran V. 2010. Interactions between xylanase and glucanase in corn-soy-based diets for broilers. Br Poult Sci 51: 246-257. doi: 10.1080/000716610037-8934

13. Dhama K, Tiwari R, Khan $R U$, Chakraborty S, Gopi M, Kartjik K, Saminathan M, et al. 2014. Growth promoters and novel additives improving poultry production and health, bioactive principles and beneficial applications: the trends and advances - a review. Int $\mathrm{J}$ Pharmacol 10: 129-159. doi: 10.3923/ ijp.2014.129.159

14. Gizzi G, Thyregod P, Von Holst C, Bertin G, Vogel K, Faurschoul-Isaksen M, Betz R, et al. 2008. Determination of phytase activity in feed: interlaboratory study. J AOAC Int 91: 259-267.

15. Gonzales E. 2004. Utilización de enzimas fibrolíticas en cabras lecheras. Evaluación de su actividad y características fermentativas in vitro. Tesis Doctoral. Barcelona, España: Univ Autónoma de Barcelona. $124 \mathrm{p}$.

16. Gracia MI, Aranibar MJ, Lazaro R, Medel P, Mateos GG. 2003. á-amylase supplementation of broiler diets based on corn. Poult Sci 82: 436-442. doi: 10.1093/ ps $/ 82.3 .436$

17. Jami E, Mizrahi I. 2012. Composition and similarity of bovine rumen microbiota across individual animals. Plos One 7: e33306. doi: 10.1371/journal.pone.0033306

18. Jiang Z, Zhou Y, Lu F, Han Z, Wang T. 2008. Effects of different levels of supplementary alpha-amylase on digestive enzyme activities and pancreatic amylase mRNA expression of young broilers. Asian Australas J Anim Sci 21: 97-102. doi: 10.5713/ajas.2008.70110

19. Kim JC, Simmins PH, Mullan BP, Pluske JR. 2004. The effect of wheat phosphorus content and supplemental enzymes on digestibility and growth performance of weaner pigs. Anim Feed Sci Technol 118: 139-152. doi:10.1016/ j.anifeedsci.2004.08.016

20. Kim M, Kim J, Kuehn LA, Bono JL, Berry ED, Kalchayanand $N$, Freetly HC, et al. 2014. Investigation of bacterial diversity in the feces of cattle fed different diets. J Anim Sci 92: 683694. doi: 10.2527/jas.2013-6841

21. Krás RV, Kessler A de M, Ribeiro AML, Henn JD, Bockor L, Sbrissia AF. 2013. Effect of dietary fiber, genetic strain and age on the digestive metabolism of broiler chickens. Braz J Poult Sci 15: 83-90.

22. Luna R, Álvarez G, Reyes M, Valverde H, Murillo de Luna G, Espinoza A, Iza $N$, Luna F. 2010. Uso de enzimas en la cría y engorde de pollos broilers en época lluviosa en las localidades de Quevedo, Salcedo y Santo Domingo de los Colorados. Cien Tecnol 3(2): 25-31.

23. Montañez O, García E, Peralta J, García J, Rojo R, Avellaneda J. 2006. Efecto de la adición de vitamina B en la digestibilidad in vitro de dietas con diferente fuente de carbohidratos. Rev Cient Zulia 16: 531-537.

24. Murphy TC, McCracken JK, McCann MEE, George J, Bedford MR. 2009. Broiler performance and in vivo viscosity as influenced by a range of xylanases, varying in ability to effect wheat in vitro viscosity. Br Poult Sci 50: 716-724. doi: 10.1080/00071660903389950

25. [NRC] National Research Council. 1994. Nutrient requirements of poultry. $9^{\text {th }}$ rev ed. [Internet]. Available in: https:/ /www.nap.edu/read/2114/chapter/1\#x

26. Olukosi OA, Cowieson AJ, Adeola O. 2008. Influence of enzyme supplementation of maize-soyabean meal diets on carcase composition, whole-body nutrient accretion and total tract nutrient retention of broilers. Br Poult Sci 49: 436445. doi: 10.1080/00071660802203732 
27. Pinheiro C, Rego JCC, Ramos TA, Silva BKR, Warpechowski MB. 2008. Digestibilidade dos nutrientes e desempenho de frangos de corte consumindo dietas formuladas com diferentes níveis de fibra e suplementadas com enzimas exógenas. Ciência Anim Bras 9: 984-996.

28. [PMAR]. 2004. Plan nacional de manejo de aguas residuales municipales en Colombia. Ministerio de Ambiente, Vivienda y Desarrollo Territorial. Bogotá, DC. 35 p. [Internet]. Disponible en: http:/ /www.minvivienda.gov.co/Documents/ Vi c e ministerio Agua/ plan_nacional_de_manejo_de_aguas_residuales_municipales_en_colombia.pdf

29. Rakotoarivonina H, Hermant B, Aubry N, Rabenoelina F, Baillieul F, Rémond C. 2014. Dynamic study of how the bacterial breakdown of plant cell walls allows the reconstitution of efficient hemicellulasic cocktails. Bioresour Technol 170: 331-341. doi: 10.1016/ j.biortech.2014.07.097

30. Santschi J, Chiquette R, Berthiaume $R$, Martineau JJ, Matte AF, Mustafa CL, Girard C. 2005. Effects of the forage to concentrate ratio on B-vitamin concentrations in different ruminal fractions of dairy cows. Canad J Anim Sci 85: 389-399.

31. Saro C, Ranilla MJ, Tejido ML, Carro $M D$. 2014. Influence of forage type in the diet of sheep on rumen microbiota and fermentation characteristics. Livestock Sci 160: 52-59. doi: 10.1016/ j.livsci.2013.12.005

32. Singh J, Dartois A, Kaur L. 2010. Starch digestibility in food matrix: a review. Trends Food Sci Technol 21: 168180. doi: 10.1016/j.tifs.2009.12.001

33. Stevenson DM, Weimer PJ. 2007. Dominance of Prevotella and low abundance of classical ruminal bacterial species in the bovine rumen revealed by relative quantification real-time PCR.
Appl Microbiol Biotechnol 75: 165-174. doi: 10.1007/s00253-006-0802-y

34. Sundset MA, Edwards JE, Cheng YF, Senosiain RS, Fraile MN, Northwood KS, Praesteng KE, et al. 2009. Molecular diversity of the rumen microbiome of Norwegian reindeer on natural summer pasture. Microb Ecol 57: 335-348. doi: 10.1007/s00248-008-9414-7

35. Svihus B, Uhlen AK, Harstad OM. 2005. Effect of starch granule structure, associated components and processing on nutritive value of cereal starch: a review. Anim Feed Sci Technol 122: 303320. doi: /10.1016/j.anifeedsci.2005.02.025

36. Svihus B. 2014. Starch digestion capacity of poultry. Poult Sci 93: 23942399. doi: $10.3382 /$ ps.2014-03905

37. Tester RF, Qi X, Karkalas J. 2006. Hydrolysis of native starches with amylases. Anim Feed Sci Technol 130: 39-54. doi: 10.1016/j.anifeedsci.2006.01.016

38. Tupayachi SG, Zea MO, Vilchez PC. 2016. Efecto de la suplementación con harina de yacón o aceite de copaiba sobre el comportamiento productivo e integridad intestinal de pollos inoculados con coccidias. Rev Inv Vet Perú 27: 475485. doi: 10.15381/rivep.v27i3.11999

39. Waite DW, Taylor MW. 2014. Characterizing the avian gut microbiota: membership, driving influences, and potential function. Front Microbiol 16: 223. doi: $10.3389 /$ fmicb. 2014.00223

40. Welkie DG, Stevenson DM, Weimer PJ. 2009. ARISA analysis of ruminal bacterial community dynamics in lactating dairy cows during the feeding cycle. Anaerobe 16: 94-100. doi: 10.1016/j.anaerobe.2009.07.002

41. Zelenka J, Ceresnakova Z. 2005. Effect of age on digestibility of starch in chickens with different growth rate. Czech J Anim Sci 50: 411-415. 